\title{
New experimental possibility to search for the ratio of a possible $T$-violating amplitude to the weak-interaction amplitude in polarized neutron transmission through a polarized nuclear target
}

\author{
V. V. Lukashevich* and A. V. Aldushchenkov ${ }^{\dagger}$ \\ St. Petersburg Institute of Nuclear Physics, Russian Academy of Sciences, Gatchina 188350, Russia \\ D. Dallman \\ CERN, Geneva, Switzerland \\ (Received 4 February 2010; revised manuscript received 3 June 2010; published 24 March 2011)
}

\begin{abstract}
This paper considers a spin-dependent neutron interaction with optical potentials (fields) from the strong interaction, the weak interaction, and an assumed $T$-violating interaction. The vector sum of these fields and their interferences determines an effective field of the target with an angular position in space due to polar and azimuthal angles. The phase of the azimuthal component is found to be the sum of two angles. The tangent of the first angle is equal to the ratio of the $T$-violating forward-scattering amplitude $D$ to the weak-interaction amplitude $C$. The quantity is of interest. The tangent of the second angle depends on the spin rotation in the residual pseudomagnetic field of the target, and it can be treated as a background effect. This paper shows that the second angle has different signs in measurements with polarized and unpolarized neutrons; thus, two measurements allow it to be compensated for. In addition, the use of the Ramsey method of separated oscillatory fields for measurement of the neutron spin rotation angle, depending on the phase of the rf field in the Ramsey cell, allows a cosine-like spectrum to be measured. This spectrum is called a phase spectrum. The phase spectra measured with polarized and unpolarized targets have a phase shift. The measurements of this phase shift with polarized and nonpolarized neutrons at a $p$-wave resonance enable the ratio $D / C$ to be isolated. We also describe the algorithm for separating the ratio $D / C$, taking into account the influence of the fringing fields of the Ramsey coil magnet and the target magnet.
\end{abstract}

DOI: 10.1103/PhysRevC.83.035501

PACS number(s): 24.80.+y, 24.70.+s, 25.40.Dn, 29.25.Pj

\section{INTRODUCTION}

The existence of an electric dipole moment (EDM) of elementary particles or atoms would testify to a simultaneous violation of parity conservation and time-reversal invariance ( $P, T$ violation). The fundamental importance of these symmetries has stimulated almost half a century of neutron EDM search since the first Ramsey experiment in 1957 [1]. The current upper limit on the neutron EDM is $d_{n} \leqslant 2.9 \times 10^{-26} e \mathrm{~cm}[2,3]$.

The search for a time-reversal symmetry violation in nuclear physics is also of fundamental significance. One of the most frequently discussed relevant experiments is the investigation of the passage of neutrons through a polarized target near the $p$-wave resonance in ${ }^{139} \mathrm{La}$. The strong dynamic enhancement of the weak interaction in this case results in a $10 \%$ asymmetry in the counting rate of neutrons $[4,5]$. Theory also predicts the enhancement of the same order of magnitude for a time-reversal-violating interaction [6].

The detection of an asymmetry caused by the timereversal violation in nuclear experiments is difficult due to a background of strong and weak interactions. In a review [7], Lamoreaux and Golub conclude that the $T$-violating amplitude in ${ }^{139} \mathrm{La}$ is unlikely to be measured in a classical polarization experiment.

\footnotetext{
*lukasche@pnpi.spb.ru

†aldu@pnpi.spb.ru

${ }^{\ddagger}$ david.dallman@cern.ch
}

According to [7], the realization of such an experiment requires an unprecedented angular accuracy (about $10^{-5} \mathrm{rad}$ ) in fixing the orientation of the neutron-polarization vector with respect to the direction of the $T$-violating field. This condition leads to the requirement that the real part of a strong-interaction field (pseudomagnetic field) should be compensated by an external magnetic field at a very high level. The angle of neutron spin rotation in a residual field should not exceed $10^{-5}$. These conditions are necessary for the measurement of $T$ violating interaction at a level $D \leqslant 10^{-4} C$ as the background effect from a weak interaction is equal to $10^{-5} C$. ( $D$ denotes the $T$-violating forward-scattering amplitude, and $C$ denotes the weak-interaction amplitude.) At the same time, in order to remove influence of an interference of a strong interaction with a weak interaction, the analyzer and polarizer efficiencies should coincide within $10^{-5}$. This is practically unattainable.

Therefore, new approaches are necessary for designing such an experiment. In this paper, we will discuss the possibility of measuring the $D / C$ ratio. The purpose of the present work is to provide a full description of the method using neutron phase spectra. The basic idea of a registration of the phase spectra is presented in Ref. [8].

As usual, the measurement of the relative value seems to be preferable to the measurements of a spin rotation in a $T$-violating field or a spin-dependent neutron absorption in this field. On the other hand, the weak-interaction field is not a source of background effects in this case. According to Ref. [9], the theoretical upper limit for the $D / C$ ratio is predicted and equal to $5 \times 10^{-4}$. It is claimed [9] that an experimental value of $D / C \leqslant 10^{-3}$ will be of importance for 
understanding the scale of the isovector binding constant of light mesons at neutron transmission.

\section{SPIN-DEPENDENT NEUTRON INTERACTION}

From a neutron spin, its wave vector, and the spin of a nucleus it is possible to assume the following correlations:

$$
B(\sigma I), C(\sigma k), C^{\prime}(k I), D(\sigma[k I]) .
$$

Traditionally, the coefficients in these correlations are the forward amplitudes for polarized neutron scattering on polarized nuclei. Keeping in mind the definition of Fermi pseudopotential, we will define each coefficient as amplitude or amplitude of interaction or interaction or corresponding field. Therefore, the first correlation corresponds to the strong spin-spin interaction $B$; the second correlation is $p$-odd and describes a weak interaction with amplitude $C$.

The third correlation is also $p$-odd and manifests in nature as a weak interaction of the neutron with the polarized nucleus. An example of this is the measurement of the asymmetry in the counting rate of neutrons moving along and against the direction of nuclear polarization of ${ }^{139} \mathrm{La}$. This asymmetry is three times higher than that arising due to field [10].

The field $D$ leads to the fourth $P, T$-violating correlation that is the subject of the present study.

Let us consider a system of coordinates in which the nuclear polarization $I$ is directed along the $z$ axis, the wave vector $k$ along the $y$ axis, and the $T$-violating field $D$ along the $x$ axis.

In the first setup, the neutrons polarized along the $z$ axis pass through the radio-frequency Ramsey coil placed in an external magnetic field. At resonance, that is, when the frequency of spin precession in an external field coincides with the radio frequency $\omega$, the neutron spin rotates by $\pi / 2$. The distribution of spins in the $x y$ plane depends on the phase of the field $\delta$ at the time of a neutron entry into the coil. The transformation of the spin components in the coil is described by the matrix (A3) shown in Appendix A. Neutron spins scan all directions in this plane, and when a neutron is detected, the phase of the rotating field of the coil is recorded. The fixed phase differs from the initial one on the product of frequency and neutron time of flight from the coil to the detector. As a result, the counting rate of neutrons is a function of this phase and is distributed in an interval from zero to $2 \pi$. It is the definition of a neutron phase spectrum. The vector sum of a $T$-violating field, a weak-interaction field, and their interference with a strong-interaction field determines the effective interaction. In the $x y$ plane, the field of effective interaction has some angular orientation called the azimuthal phase of the interaction. The counting rate of neutrons is different for neutrons with spins directed along and against the effective field. Therefore, a phase spectrum has an oscillating form. The target in this case plays the role of an analyzer. This experimental configuration will be designated polarizer, coil, target, and detector (PCTD).

For the second measurement, which is necessary for eliminating the background effects, the target is a polarizer, and the neutrons impinging on it are not polarized. The absorption of neutrons with spins directed along and against the effective field is also different, and after passage through the target, the neutron beam gets two polarization components. The first is an azimuthal component $p_{a z i}$ (in the $x y$ plane), and the second one is a component $p_{z}$ by virtue of correlation $B(\sigma I)$. Then neutrons pass through the Ramsey coil, turning these components at $\pi / 2$, the analyzer, and hit the detector. The analyzer is transparent only for the first component. This is the configuration target, coil, analyzer, and detector (TCAD).

Calibration spectra are measured with the target polarization switched off for each configuration. In this case, either the direction or the phase of the weak-interaction field in the target is known. This enables the phase scale to be calibrated and the phase shift of the field with a polarized target to be found.

\section{NEUTRON SPECTRA AND PHASE SHIFTS}

The following expression describes a spectrum with initially polarized neutrons (configuration PCTD):

$$
N_{p c t}(\delta, t)=\frac{1}{2} \operatorname{Tr}\left(U_{t} U_{c t} U_{c} \rho_{p} U_{c}^{\dagger} U_{c t}^{\dagger} U_{t}^{\dagger}\right)=\frac{1}{2} \operatorname{Tr}\left(\rho_{a}^{\prime}\right),
$$

where the subscripts on the evolution operators correspond to the coil $(c)$, the coil-target distance $(c t)$, and the interaction with the target $(t)$, and $\rho_{p}=\frac{1}{2}\left(1+\boldsymbol{\sigma} \boldsymbol{p}_{p}\right)$ is the spin-density matrix of the polarizer, normalized to the unit neutron flux.

The evolution operator for some field $\boldsymbol{b}$ has the following form:

$$
U=\exp \left(\frac{-i t}{2}(\boldsymbol{\sigma} \boldsymbol{b})\right),
$$

where $\sigma$ is Pauli matrix and $t$ is a neutron time of flight in the field $\boldsymbol{b}$.

The new spin polarization vector $\boldsymbol{p}_{a}^{\prime}$ determines the matrix $\rho_{a}^{\prime}$. This vector is the analyzing power of a target.

The corresponding expressions for the configuration TCAD are

$$
N_{t c a}(\delta, t)=\frac{1}{2} \operatorname{Tr}\left(\rho_{a} U_{c} U_{t c} U_{t} U_{t}^{\dagger} U_{t c}^{\dagger} U_{c}^{\dagger}\right)=\operatorname{Tr}\left(\rho_{a} \rho_{p}^{\prime}\right) .
$$

Here $\rho_{a}=\frac{1}{2}\left(1+\boldsymbol{\sigma} \boldsymbol{p}_{a}\right)$ is the density matrix of the analyzer, and the symbols $a$, $c$, and $t$ refer to the analyzer, coil, and target, as mentioned above. Being the polarization power of a target, the vector $\boldsymbol{p}_{p}^{\prime}$ is included in the definition of the matrix $\rho_{p}$.

The expressions for these spectra have the following form: $N_{p c t(t c a)}(\delta, t)=\frac{1}{2} \exp (-A t)\left[N_{0}+p_{p(a)} p_{a(p)}^{\prime} \cos \left(\alpha_{p c t(t c a)}\right)\right]$.

In Eq. (3) the exponent describes the neutron spin-independent absorption in the target, where $N_{0}$ takes into account the change in the normalization of the density matrix due to the absorption of neutrons; $t$ is the time of flight through the target. The quantity $p_{a(p)}^{\prime}=\sqrt{p_{x a(p)}^{\prime}+p_{y a(p)}^{\prime}}$ is the azimuthal component of the analyzing (polarizing) power of the target, which has the angular orientation (see Fig. 1) $\beta_{a(p)}=\operatorname{arctg}\left(p_{y a(p)}^{\prime} / p_{x a(p)}^{\prime}\right)$. The angle $\alpha$ in Eq. (3) is the angle between vectors $\boldsymbol{p}$ and $\boldsymbol{p}^{\prime}$, with this angle having a different meaning in the two cases. The expression for this angle is calculated with the help of matrix (A3) of Appendix A.

Here $\alpha_{p c t}=\delta+\alpha_{t l}-\beta_{a}-\pi / 2$, where $\alpha_{t l}$ is the angle of spin rotation in the left fringing field of the target magnet and $\delta$ is a phase of a rotating field at the moment of an exit of a neutron from the coil. 


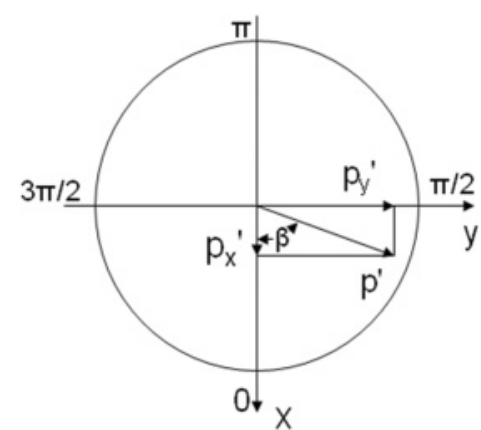

FIG. 1. The phase diagram of the azimuthal component of neutron spin interaction with a target.

For the TCAD mode this angle is $\alpha_{t c a}=\delta-\beta_{p}-\alpha_{t r}+$ $\pi / 2$. Here $\alpha_{t r}$ is the angle of spin rotation in the right fringing field of the target magnet.

Let us assume that the gyromagnetic ratio for the neutron is equal to 1 . In this case, the intensity of the magnetic field, as well as the fields in the target, and the precession frequency have the same dimension.

In calculating expressions (1) and (2), according to Ref. [7], we introduce a vector of an effective field $\boldsymbol{b}$ with the following components:

$$
b_{x}=\frac{p_{t}}{2} D[k I], \quad b_{y}=\frac{C}{2} k, \quad b_{z}=\frac{p_{t}}{2} B I .
$$

Later, new notation will be used:

$$
\boldsymbol{b}^{\prime}=\boldsymbol{b} \frac{\sin (q t)}{q}, \quad q=\sqrt{\boldsymbol{b} \cdot \boldsymbol{b}} .
$$

The solution of the Eqs. (1) and (2) provides new expressions for the analyzing and polarizing power of the target:

$$
\begin{aligned}
& p_{x a(p)}^{\prime}=2 \operatorname{Im}\left(b_{x}^{\prime *} \cos q t\right) \pm 2 \operatorname{Im}\left(b_{y}^{\prime} b_{z}^{\prime *}\right), \\
& p_{y a(p)}^{\prime}=2 \operatorname{Im}\left(b_{y}^{\prime *} \cos q t\right) \pm 2 \operatorname{Im}\left(b_{z}^{\prime} b_{x}^{\prime *}\right), \\
& p_{z a(p)}^{\prime}=2 \operatorname{Im}\left(b_{z}^{\prime *} \cos q t\right) .
\end{aligned}
$$

The second terms in Eqs. (4) and (5) represent an interference of fields $C$ and $D$ with the field of strong interaction, respectively. Expression (6) would also contain such a term, but because of the resonant behavior of $C$ and $D$, this term is equal to zero. The terms in Eqs. (4), (5), and (6) are shown in more detail in Appendix A. In relations (4) and (5) the upper signs correspond to the target playing the role of an analyzer. The lower signs correspond to the case where nonpolarized neutrons hit the target and the target is a polarizer.

The value $N_{0}$ in Eq. (3) is

$$
N_{0}=\frac{|b|^{2}}{|q|^{2}}|\sin (q t)|^{2}+|\cos q t|^{2} .
$$

As the target is in an external magnetic field, the residual field is equal to $\operatorname{Re} 2 b_{z}=\operatorname{Re} B \Rightarrow p_{t} \operatorname{Re} B-H$, where $p_{t}$ is the target polarization and $\operatorname{Re} B$ is the nuclear constant.

Now we can find the angular orientation of the analyzing (polarizing) power of the target $\beta_{a(p)}$, supposing that the interaction $b_{x}$ has the same resonant behavior as the interaction $b_{y}$.

$$
\operatorname{tg} \beta_{a(p)}=p_{y a(p)}^{\prime} / p_{x a(p)}^{\prime}=\operatorname{tg}\left[\pi / 2-\left(\beta_{1} \pm \beta_{2}\right)\right],
$$

where

$$
\begin{aligned}
& \operatorname{tg} \beta_{1}=-\operatorname{Im}\left(b_{z}^{\prime} b_{x}^{\prime *}\right) / \operatorname{Im}\left(b_{y}^{\prime} b_{z}^{\prime *}\right)=\operatorname{Im} b_{x} / \operatorname{Im} b_{y}, \\
& \operatorname{tg} \beta_{2}=\operatorname{Im}\left(b_{y}^{\prime} b_{z}^{\prime *}\right) / \operatorname{Im}\left(b_{y}^{\prime *} \cos q t\right) .
\end{aligned}
$$

Here we use expressions (4) and (5) and the equality

$$
\frac{\operatorname{Im}\left(b_{x}^{\prime *} \cos q t\right)}{\operatorname{Im}\left(b_{y}^{\prime *} \cos q t\right)}=\frac{\operatorname{Im} b_{x}}{\operatorname{Im} b_{y}},
$$

which follows from

$$
\frac{\operatorname{Re} b_{x}}{\operatorname{Im} b_{x}}=\frac{\operatorname{Re} b_{y}}{\operatorname{Im} b_{y}}
$$

and expressions (B1)-(B3) of Appendix B. The latter equality reflects the properties of real and imaginary amplitudes in $p$-wave resonance.

From these calculations, one can conclude that the sum of two angles $\beta_{a}$ and $\beta_{p}$ defines the doubled value of the ratio of $T$-violating amplitude to weak-interaction amplitude. However, measuring the ratio is possible if the value $p_{a(p)}^{\prime}$ in Eq. (3) is not very small.

The hierarchy of the spin-dependent interactions is $B \gg$ $C \gg D$, and each subsequent interaction is between three and four orders of magnitude less than the previous one. Let us consider the compensation of the pseudomagnetic field when the following condition is satisfied:

$$
\operatorname{Re} B \approx \operatorname{Re} 2 q \gg \operatorname{Im} B, \operatorname{Im} 2 q, C, D .
$$

This condition is easy to meet because the pseudomagnetic field (for example, in ${ }^{139} \mathrm{La}$ ) has a scale about some kilogauss and the field of weak interaction is four orders less.

Then expressions (4)-(6) become

$$
\begin{aligned}
p_{x a(p)}^{\prime} & \approx-a p_{t} \operatorname{Im} D t \pm d \operatorname{Im} C t, \\
p_{y a(p)}^{\prime} & \approx-a \operatorname{Im} C t \mp d p_{t} \operatorname{Im} D t, \\
p_{z}^{\prime} & \approx-\operatorname{Im} B .
\end{aligned}
$$

Here the factor $a=\sin (\operatorname{Re} B t) / \operatorname{Re} B t$ decreases the influence of fields $C$ and $D$ at the polarizing (analyzing) power of the target. This factor depends on the precession of the spin in the residual pseudomagnetic field. The factor

$$
d=\frac{2 \sin ^{2}(\operatorname{Re} B t / 2)}{\operatorname{Re} B t}
$$

shows how the spin precession changes the interference term.

According to the hierarchy of forces, there are two limiting cases: $q t \ll 1$ and $q t \geqslant 1$. In the former case, when interference contributions are small, $d \approx \operatorname{Re} B t / 2$ and the measurement of field $D$ is made in the direction of the $x$ axis. In most discussions of the separation of the $T$-violating field (see Ref. [7]), this limiting case has been studied more often. We note that in the case of full compensation of the pseudomagnetic field the interference contribution does not disappear entirely because strong interaction still contains an imaginary part.

The effects of fields $C$ and $D$ are minimal when $q t \geqslant 1$, but in this case, the interference contribution remains significant. The dependence of $a$ and $d$ on the angle of spin rotation in a residual field is shown in Fig 2. The equality 


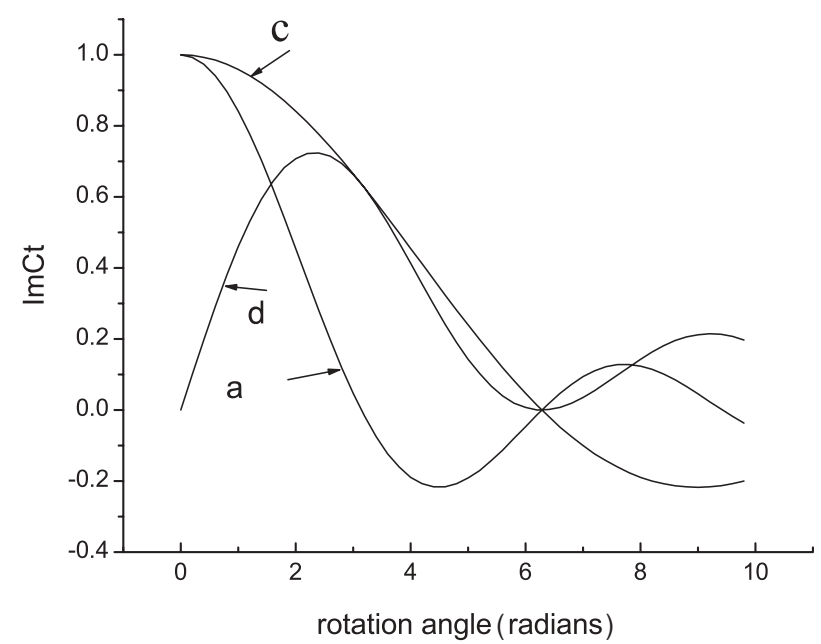

FIG. 2. Dependence of the quantities $a, d$, and $c$ on the angle of spin rotation in a residual pseudomagnetic field.

$\operatorname{Re} B t=t g(\operatorname{Re} B t / 2)$ corresponds to the positions of the interference maxima.

At angle $\operatorname{Re} B t=\pi$, when the factor $a=0$, the action of the basic fields on the spin of the neutron is minimal, and the spins interact with the interference fields. The value $p^{\prime}$ is equal to

$$
p^{\prime}=\frac{2 \operatorname{Im}\left(b_{y}^{\prime *} \cos q t\right)}{\cos \beta_{2}} \sqrt{1+p_{t}\left(\operatorname{Im} b_{x} / \operatorname{Im} b_{y}\right)^{2}} \approx c \operatorname{Im} b_{y},
$$

where $c=\sin (B t / 2) /(B t / 2)$. The approximate value takes place at the realization of condition (9). The dependence of factor $c$ on the angle of spin rotation is also shown in Fig. 2. It is seen that in the interval $-\pi<B t<\pi$, parameter $c$ is changed from $2 / \pi$ to unity. This interval determines an allowable degree of compensation of a pseudomagnetic field in a real experiment. Outside this interval, a spin rotation decreases the amplitude of cosine in a neutron spectrum (3), and it is impossible to observe the oscillation of the spectrum. The counting rate in expression (3) is maximal if the angular position of the neutron spin coincides with the phase $\beta$. However, the angular position of the neutron spin is not observed, so to find the field phase $\beta$, it is necessary to calibrate the phase scale in each case. This can be done in the following way. The polarization of the target and the target magnetic field are switched off. The fields $B$ and $D$ are proportional to the target polarization; thus, they disappear, and there remains only the weak-interaction field $C$ in the target. This field is along the $y$ axis and has, according to Fig. 1, a phase $\pi / 2$. Concerning the calibration spectrum, the phase shift for polarized neutrons becomes $\Delta_{p c t}=\beta_{a}+\alpha_{t l}$, as shown in Fig. 3. For the configuration TCAD this phase shift will be $\Delta_{t c a}=\beta_{p}-\alpha_{t r}$. Here $\alpha_{t l(r)}$ is a spin rotation angle in the left (right) fringing field of the target magnet. The sum of phase shifts $\Delta_{p c t}$ and $\Delta_{t c a}$ is

$$
\Delta=2 p_{t} \frac{\operatorname{Im} D}{\operatorname{Im} C}+\alpha_{t l}-\alpha_{t r} .
$$

The difference of phases due to the precession of the spin in the fringing fields of the target magnet can result in a systematic

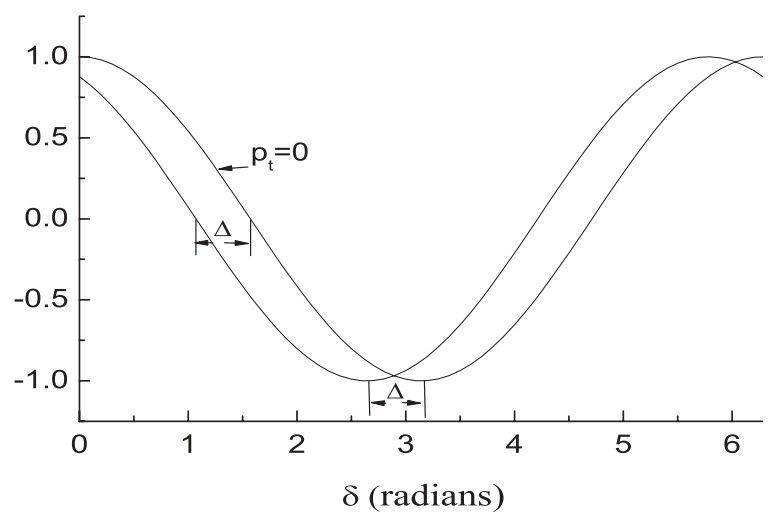

FIG. 3. Phase shift of a neutron spectrum concerning a calibrating spectrum when the field of the target magnet and the polarization of the target are switched off.

error, and to eliminate it, it is necessary to carry out several rounds of measurements. In these cases, the polarization of the target is changed in such a manner that the angle of spin precession in a residual field approximately ranges in interval from $\pi$ to $-\pi$. By virtue of the linear dependence of $\Delta$ on $p_{t}$, we have

$$
\frac{\partial \Delta}{\partial p_{t}}=2 \frac{\operatorname{Im} D}{\operatorname{Im} C} .
$$

Let us discuss now some possible reason for the systematic errors responsible for the background effect at the measurement of $D / C$. Nonparallelity of a polarizer (analyzer) axis with a direction of an external magnetic field of a Ramsey cell is likely to give rise to such errors. If the polarizer (analyzer) has small polar $\theta$ and azimuthal $\varphi$ angles of orientation, an additional expression appears in the spectrum (3) caused by spin movement in the Ramsey cell: $\Delta N=p_{a(p)} p_{z}^{\prime} \theta \sin (\delta-\varphi)$. The oscillating part of the spectrum (3) has the following view:

$$
\begin{aligned}
& p p^{\prime}\left[c \cos \alpha+\theta\left(p_{z}^{\prime} / p^{\prime}\right) \sin (\delta-\varphi)\right] \\
& \quad \approx p p^{\prime}\left[\cos \alpha+\theta\left(\operatorname{Im} b_{z} / c \operatorname{Im} b_{y}\right) \sin (\delta-\varphi)\right] .
\end{aligned}
$$

It can be immediately seen that there are two limiting cases at the point $\alpha=\pi / 2$, defining a possible interval of the systematic shift $\Delta_{\text {sys }}: 0<\Delta_{\text {sys }}<\theta \operatorname{Im} B / c \operatorname{Im} C$. The first takes place at $\delta-\varphi=\pi / 2-\alpha$, and the second takes place at $\delta-\varphi=\alpha$. However, it is impossible to determine the allowable value of an angle $\vartheta$ exactly because the imaginary part of strong interaction is not known.

Finally, we will estimate the sensitivity of the described algorithm determining the phase shift $\beta_{1}$. For this purpose, we note that expression (7) has another approximate form,

$$
N_{0}=\frac{|b|^{2}}{|q|^{2}}|\sin q t|^{2}+|\cos q t|^{2} \approx \cosh (\operatorname{Im} q t) \approx 1,
$$

due to the weak absorption of neutrons in the spin-dependent fields of the target. Thus, the following expressions describe the experimental spectra for both the nonpolarized and polarized targets, respectively:

$$
n_{1}=\frac{n}{2 \pi}(1+A \cos \alpha), \quad n_{2}=\frac{n}{2 \pi}[1+A \cos (\alpha+\chi)] .
$$


In these expressions, $n_{i}$ is the number of detected neutrons (identical in both spectra), and the asymmetry arising from the influence of a weak-interaction field is $A \approx 0.1$.

The situation is similar to the measurement of $\gamma$-line energy in nuclear spectroscopy by the center-of-gravity shift relative to the reference line.

The root-mean-square deviation of the phase shift is equal to

$$
\sigma(\chi)=\frac{\pi \sqrt{2}}{A \sqrt{n}} .
$$

For $\chi$ we use the value $\chi \leqslant 5 \times 10^{-4}$ from Ref. [9]. Then the statistics necessary for measuring the phase shift to $30 \%$ is $n \approx 2 \times 10^{10}$. It takes a week to collect such a statistic at a neutron flux of $10^{5}$ in the region of $1 \mathrm{eV}$. It means that the prospective experiment is quite realistic.

\section{CONCLUSIONS}

The results obtained above show a preference for a separation of the $T$-violating field in the angle region from $\pi$ to $-\pi$ for the spin rotation in a residual pseudomagnetic field.

The ratio $\operatorname{Im} D / \operatorname{Im} C$, representing part of a azimuthal phase of an effective field of a target, is separated from spectra measured with polarized and unpolarized neutrons. In each case, the phase scale is calibrated with respect to the weak interaction.

It is shown that the field of weak interaction, the pseudomagnetic field, and their interference cannot be responsible for masking effects.

Since the problem is reduced to measurements of phase shifts, the strict equality of both polarizer and analyzer efficiencies is not required.

It is shown that the fringing fields of the Ramsey coil magnets are taken into account by calibrations of the phase scale and do not result in systematic errors. The algorithm described also allows eliminating the influence of the fringing fields of the target magnet on the result of the measurement of the ratio $\operatorname{Im} D / \operatorname{Im} C$.

It is natural that before measuring the $T$-violating amplitude the values of the real and imaginary parts of strong interaction should be determined in the area of a $p$-wave resonance.

\section{ACKNOWLEMDGMENT}

The authors are grateful to Dr. A. P. Serebrov for helpful discussions.

\section{APPENDIX A}

The evolution operator for a Ramsey coil at resonance, that is, when the frequency of spin precession in the external field $\omega_{0}$ coincides with the frequency $\omega$ of a rotating field, is

$$
U_{c}=\exp \left(-i \omega t \sigma_{z}\right) \exp \left[-i \omega_{1} t(\boldsymbol{\sigma} \cdot \boldsymbol{n})\right] .
$$

Here $\omega_{1}$ is the frequency of spin precession in a field equal to the amplitude of the rotating field, $\sigma$ is the Pauli matrix, and $\boldsymbol{n}$ is the unit vector of the field direction at the moment of the neutron coming into the coil, determined by the phase of the field $\delta: n_{x}=\cos \delta, n_{y}=\sin \delta$, and $n_{z}=0$.

This evolution operator transforms the components of a vector of polarization $\boldsymbol{p}_{0}$ as follows:

$$
\left(\sigma p_{1}\right)=U_{c}\left(\sigma p_{0}\right) U_{c}^{\dagger} \text {. }
$$

The equality $\left(\boldsymbol{p}_{1}\right)=M_{c}\left(\boldsymbol{p}_{0}\right)$ defines a three-dimensional matrix of polarization vector transformation. This matrix is

$$
M_{c}=\left(\begin{array}{ccc}
\cos (\omega t+\delta) \cos \delta & \cos (\omega t+\delta) \sin \delta & \sin \omega_{1} t \sin (\omega t+\delta) \\
+\cos \omega_{1} t \sin \delta \sin (\omega t+\delta) & -\cos \omega_{1} t \cos \delta \sin (\omega t+\delta) & \\
\sin (\omega t+\delta) \cos \delta & \sin (\omega t+\delta) \sin \delta & -\sin \omega_{1} t \cos (\omega t+\delta) \\
-\cos \omega_{1} t \sin \delta \cos (\omega t+\delta) & +\cos \omega_{1} t \cos \delta \cos (\omega t+\delta) & \\
-\sin \omega_{1} t \sin \delta & \sin \omega_{1} t \cos \delta & \cos \omega_{1} t
\end{array}\right)
$$

For a neutron with energy $E_{0}$ (a maximum $p$ resonance) an angle of spin rotation is $\omega_{1} t_{0}=\pi / 2$.

\section{APPENDIX B}

Here we show in detail the expressions for the quantities in relations (4), (5), and (6).

$$
2 \operatorname{Im}\left[b_{i}^{* *} \cos (q t)\right]=\frac{\sin (2 \operatorname{Re} q t)}{|q|^{2}}\left(\operatorname{Re} b_{i} \operatorname{Im} q-\operatorname{Re} q \operatorname{Im} b_{i}\right)-\frac{\sinh (2 \operatorname{Im} q t)}{|q|^{2}}\left(\operatorname{Re} b_{i} \operatorname{Re} q+\operatorname{Im} b_{i} \operatorname{Im} q\right)
$$

Here $i=x, y, z$.

$$
\begin{aligned}
& 2 \operatorname{Im}\left(b_{y}^{\prime} b_{z}^{\prime *}\right)=\frac{2}{|q|^{2}}\left[\sin ^{2}(\operatorname{Re} q t)+\sinh ^{2}(\operatorname{Im} q t)\right]\left(\operatorname{Re} b_{z} \operatorname{Im} b_{y}-\operatorname{Re} b_{y} \operatorname{Im} b_{z}\right) . \\
& 2 \operatorname{Im}\left(b_{z}^{\prime} b_{x}^{\prime *}\right)=\frac{2}{|q|^{2}}\left[\sin ^{2}(\operatorname{Re} q t)+\sinh ^{2}(\operatorname{Im} q t)\right]\left(\operatorname{Re} b_{x} \operatorname{Im} b_{z}-\operatorname{Re} b_{z} \operatorname{Im} b_{x}\right) .
\end{aligned}
$$


[1] J. Smith, E. Purcell, and N. Ramsey, Phys. Rev. 108, 120 (1957).

[2] C. Baker et al., Phys. Rev. Lett. 97, 131801 (2006).

[3] C. Baker et al., Phys. Rev. Lett. 98, 149102 (2007).

[4] V. P. Alfimenkov et al., Nucl. Phys. A 398, 93 (1983).

[5] J. D. Bowman et al., Phys. Rev. Lett. 65, 1192 (1990).

[6] V. P. Gudkov, Phys. Rep. 212, 77 (1992).
[7] S. K. Lamoreaux and R. Golub, Phys. Rev. D 50, 5632 (1994).

[8] V. V. Lukashevich and A. V. Aldushchenkov, Yad. Fiz. 70(4), 714 (2007).

[9] I. S. Towner and A. C. Hayes, Phys. Rev. C 49, 2391 (1994).

[10] V. P. Alfimenkov et al., Yad. Fiz. 59(11), 1929 (1996). 